\title{
Application of thermography in surgical curing of hand oversweating
}

\author{
by K. Goździuk ${ }^{1}$, P. Baranowski ${ }^{3}$, W. Mazurek ${ }^{3}$, O. Kalisz ${ }^{2}$, T. Wolski ${ }^{4}$, M. Sawicki ${ }^{1}$, A.Rojowski ${ }^{1}$ \\ ${ }^{1}$ Department and Clinic of Surgery of Chest of Medical University in Lublin, Poland \\ ${ }^{2}$ II Clinic of Ophthalmology of Medical University in Lublin, Poland \\ ${ }^{3}$ Institute of Agrophysics, Polish Academy of Sciences, Lublin, Poland \\ ${ }^{4}$ Department and Unit of Pharmacognozy of Medical University in Lublin, Poland
}

\begin{abstract}
In this paper we made a trial to apply thermography for controlling the course of sympathectomy surgery and for evaluation of hand sweating intensity decrease in particular stages of surgery. The patients were examined with thermogarphy before, during and 2 days after the surgical treatment. Shortly after cutting successive thoracic ganglia, patients' hand temperature increased by several degrees. The temperature changes on hand surface during surgery revealed that cutting of the fifth ganglion has the least effect on diminishing of oversweating symptoms. In post surgery period distribution of body temperature was for all patients close to temperature distribution of healthy volunteers.
\end{abstract}

\section{Introduction}

Current medical management is directly dependent on many diagnostic imaging methods. These methods are especially useful in operative specialties. Several technologically advanced methods are available at the current level of knowledge and medical technique: computed tomography (CT), magnetic resonance imaging (MRI), positron emission tomography (PET), radioisotopic methods and infrared imaging, which becomes more and more popular. Examination of the human body by measurement of the temperature is as old as medicine. Current increase in interest in use of infrared imaging for detecting and monitoring diseases of different sites of human body is caused by a few reasons. They are: improvement of the diagnostic instrument which is thermographic camera, non-invasiveness of the examination, and very low costs of application of the method. Medical benefits from using infrared imaging are evident, which we will try to show in this report. This technique shall not be considered as a rival to widely known medical examination techniques, but as their precious completion.

Thoracic sympathectomy is a procedure consisting in elective surgical destruction of certain thoracic sympathetic chain segments to switch off the stimulation of elected body functions. It has been in use as a treatment procedure for over a hundred years, but it is widely used only after introduction to surgery of videothoracoscopic methods, which decreased the risk of postoperative complications.

The aim of the study: We tried to assess the effectiveness of palmar hyperhydrosis surgical treatment with use of bilateral thoracic sympathectomy with low-invasive videothoracoscopic technique.

\section{Methods}

\subsection{Anatomical and physiological data}

Sympathetic trunk is the pair of ganglionated longitudinal cords of the sympathetic nervous system of which one is situated on each side of the spinal column that runs from the base of the skull to the coccyx. Preganglionic fibers (white rami communicans) run from the spinal cord to the sympathetic ganglia in ventral nerve roots, and grey rami communicans run from the symphatetic ganglia to the spinal nerves. The thoracic part of the sympathetic trunk consists of 10-12 thoracic ganglia. The first thoracic ganglion often connects with the inferior cervical ganglion forming the stellate ganglion. Stimulation of the sympathetic nervous system causes papillary dilation, increased sweat secretion, contraction of digestive tract sphincters, relaxation of the gall bladder, urethers and urinary bladder, acceleration of the heart rate and force of contraction, vasoconstriction in most of vasels, increase in blood pressure and metabolism [4].

\subsection{Description of surgery}

The procedure is performed in general anaesthesia with separate ventilation of each lung through a double lumen endotracheal tube. At bilateral procedure the patient should be placed on the operating table in a semireclining position with both arms perpendicular to the trunk and the head tilted back. Two troacars $5 \mathrm{~mm}$ in diameter are inserted into the pleural cavity on the operated side through the III and V intercostal space in the midaxillary line. After inserting the video-camera through the lower troacar, the sympathetic trunk located along the vertebral column about $1,5 \mathrm{~cm}$ from the heads of the ribs should be identyfied under the parietal pleura. Depending on the range of hyperhydrosis, adequate ganglia should be destructed: ganglion II - for palms, ganglia III and IV - for arm pits, and branches from ganglion II - for the face. Stellate ganglion sholuld never be damaged, because it leads to Horner's syndrome with typical face deformation. 


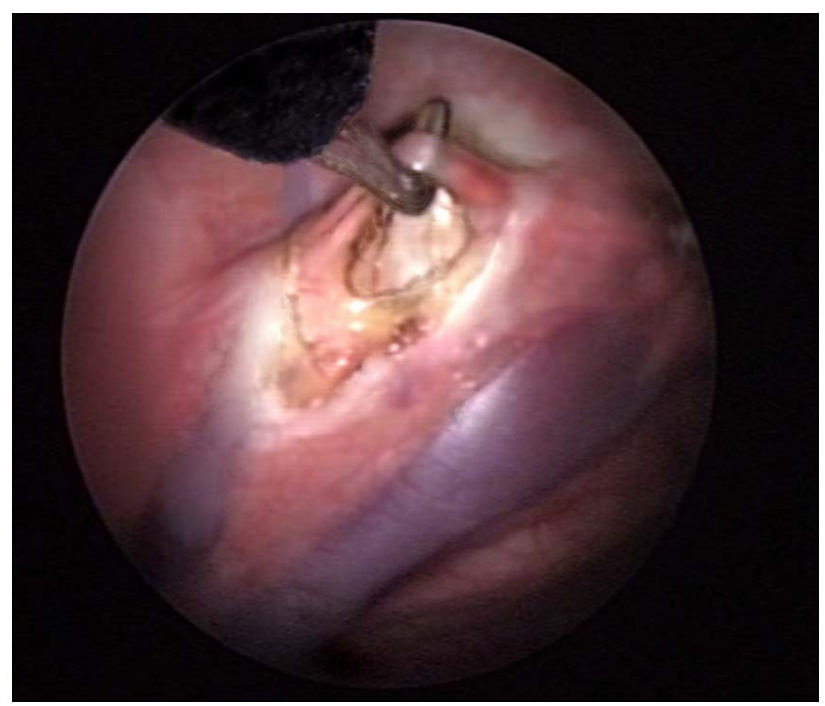

Fig. 1. The moment of nerve cutting during sympathectomy surgery

\subsection{Procedure of thermographic measurement}

Vegetative nervous system is responsible for contractibility of smooth muscles, what manifests with intensity of blood flow through skin and other organs. Therefore, it has direct impact on dynamics of circulation and processes of body thermoregulation $[2,3,6]$. In case of oversweating the domination of the sympathetic system over parasympathetic system leads to increased production and exudation of sweat in spite of the absence of thermic and climatic factors of the surrounding regulating this process in physiological manner. It was expected that thermographic map of body created before and after surgery could explain many processes connected with pathology of vegetative system functioning $[1,7]$.

The thermographic examination was performed with the use of VIGOcam v50 by VIGO System SA, working in the range of $8-14 \mu \mathrm{m}$. The thermographic system is equipped with CCD camera working in visible range and a laser sight used to localize central point of registration. This system enables to measure the radiation temperature with radiometric sensitivity of $0,08^{\circ} \mathrm{C}$. Except from the standard software "THERM" all statistical analyses and thermal image processing were performed using freeware "ImageJ" [5]. Radiation temperature measurements were conducted in stable and controlled external conditions. It was done by thermo-stabilisation of the laboratory and installation of air temperature, air humidity and pressure sensors. All the measurements were performed in the temperature of $25^{\circ} \mathrm{C}$ under relative air humidity of $60 \%$ in daily light. The measurements were conducted from the distance of $1 \mathrm{~m}$. It was assumed on the base of literature data that emissivity coefficient of skin is equal 0,99 .

A group of 5 patients suffering from skin oversweating were qualified to have thermographic examination. The clinic manifestation of this disease was mainly localized in the area of hands. The patients were examined with thermogarphy one day before, during and two days after the surgical treatment, which consisted in performing chest sympathecthomy with the use of lightly invasive method of video thoracoscopy. The thermographic examination of patients before and after surgery included taking thermograms of inner and outer parts of hands, armpits and feet. During the surgery the studied palm was fixed outside the operating table and camera was situated directly beneath the palm looking perpendicularly up on it from the distance of about $1 \mathrm{~m}$. The surgery of each patient included left and right side of a chest at a time, therefore both palms were the subject of thermographic examination during thoracic ganglia cutting. To check the influence of particular ganglia cutting on postsurgery effect, the order of ganglia cutting was different for particular cases: II, III, IV, V or V, IV, III, II.

Thermographic images were compared applying special computer analysis procedure. To analyze obtained thermograms, two areas were selected on the palm. One of them was a point indicator on the middle finger and the second was a circle in the middle of the palm. The registration was performed with the rate of two frames per second. The average time of registration of one hand during the surgery was about 6 minutes. The courses of temperature changes directly before and after cutting ganglia were examined. The air temperature and relative humidity in operating room were monitored with the use of LAB-EL system (LB705 model) with the time interval of two minutes.

\section{Results}

Thermographic examination of palm temperature before and after the surgery revealed considerable changes of temperature distribution (figure 2). For the studied causes the increase of average palm temperature from $3,4^{\circ} \mathrm{C}$ to $9,2^{\circ} \mathrm{C}$ was observed two days after the surgery. It was the result of decrease of sweat exudation 
and smaller energy loss used as latent heat of vaporization. Another result of the surgery was smaller differentiation of temperature distribution on the palms of all studied cases.
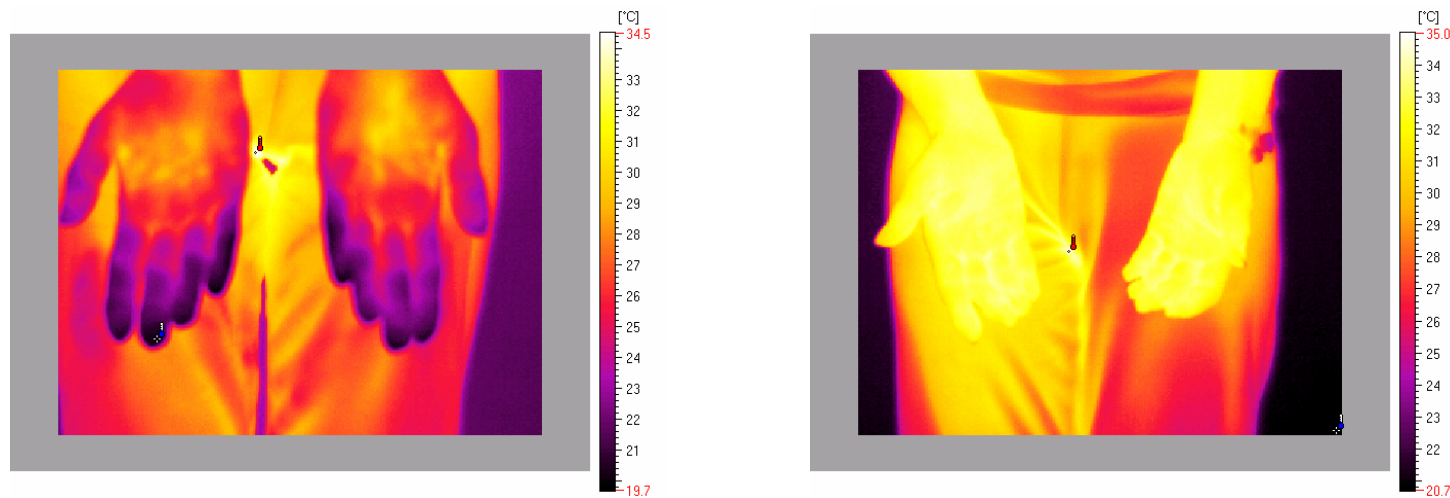

Fig. 2. Thermograms of inner side of patient's palms: one day before (left) and two days after (right) the sympathectomy surgery

The sequential registration of palm temperature enabled to monitor the rate of the decrease of sweating intensity after cutting particular thoracic ganglia. In figure 3 two thermograms of one patient's hand is presented at the moment of second ganglion cutting and two minutes later. In both selected areas considerable increase of temperature was observed reaching $5^{\circ} \mathrm{C}$ in the middle finger part and $2.5^{\circ} \mathrm{C}$ in the middle of the palm.

The intensity of temperature increase was different after cutting various ganglia and it also depended on the order of ganglia cutting. For the order from II to V ganglia the highest temperature increase was stated after cutting II and III ganglia. The example of temperature changes after cutting particular ganglia is presented in figure 4 .
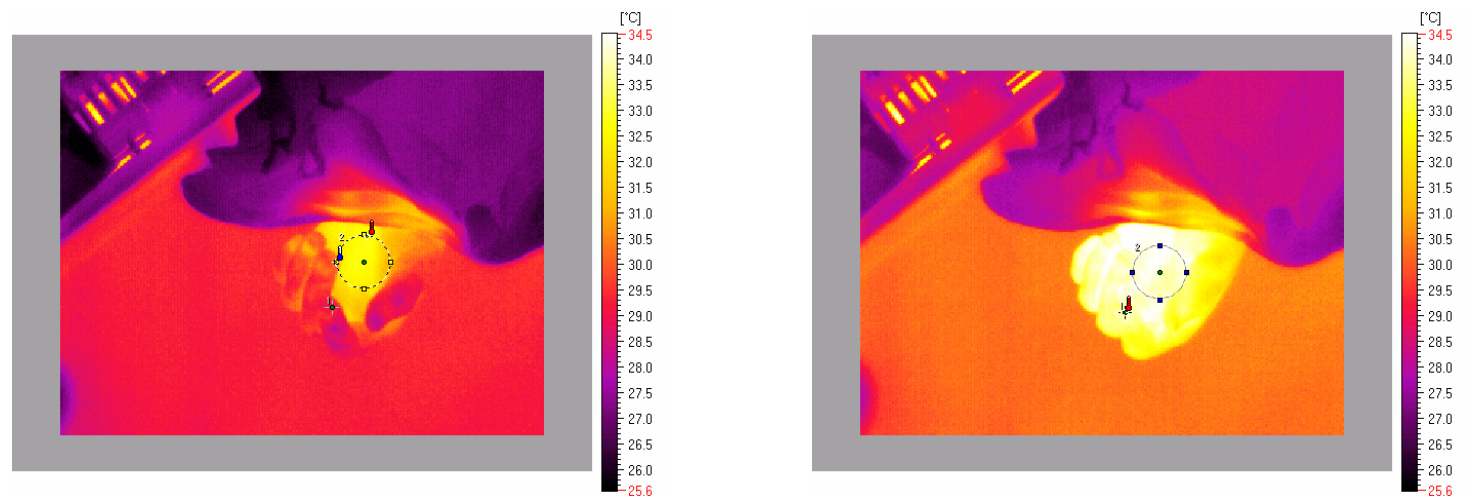

Fig. 3. Thermograms of inner side of patient's palm: at the moment of cutting (left) and two minutes after cutting (right) the second ganglion during sympathectomy surgery

It results from figure 4 that for the studied patient the increase of temperature after cutting the second ganglion reached $4.2^{\circ} \mathrm{C}$ in the period of 2.5 minutes. After cutting third ganglion the temperate increased by $3^{\circ} \mathrm{C}$ while after cutting the fourth ganglion it increased by $2.2^{\circ} \mathrm{C}$. Regardless of the order of ganglia cutting for all studied patients the temperature increase after cutting the fifth ganglion was very small (less than $0.8^{\circ} \mathrm{C}$ ) and it confirmed expectation that cutting this ganglion the least important for diminishing the sweating symptoms. After performing two operations it was decided that in further surgeries cutting of this ganglion should be omitted. It is a very important conclusion coming from thermographic studies that cutting II, III and IV ganglion is satisfactory to stop oversweating of hands. This result enabled to simplify and shorten the operation procedure. 


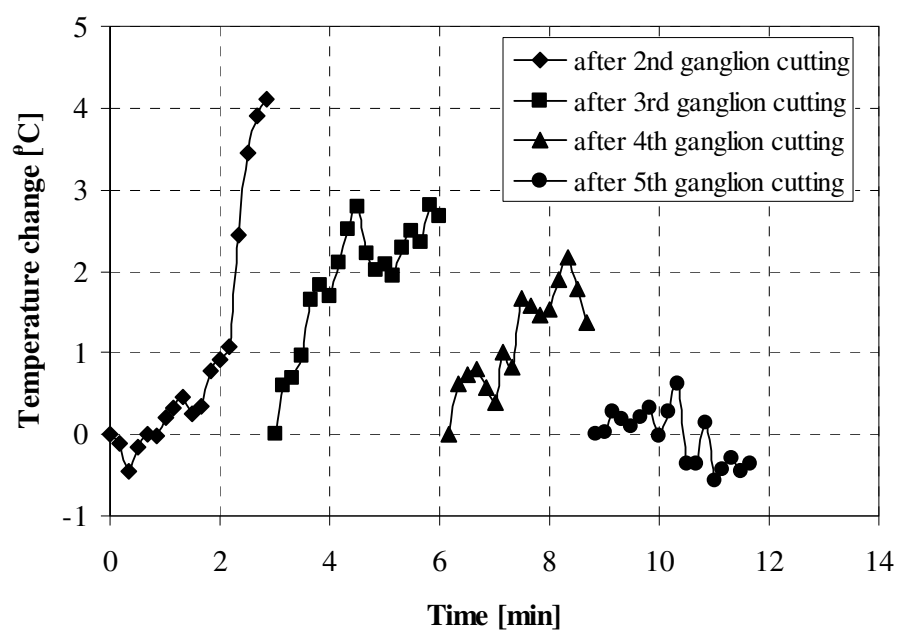

Fig. 4. Temperature changes in succeeding stages of sympathectomy surgery

For all the studied patients the statistical analysis of palm temperature changes in particular stages of surgery was performed. In figures 5 and 6 the average temperature increases after cutting particular ganglia applying inversed order of cutting are presented jointly with analysis of average air temperature changes. It results from figure 5 that for the order of cutting: II, III, IV, V, the highest average temperature increase occurred after cutting second and third ganglia, reaching $3.5^{\circ} \mathrm{C}$ and $1.5^{\circ} \mathrm{C}$, respectively. It is worth to notice that the range of temperature change after cutting the third ganglion for this order of cutting is very large. Considerably smaller increases of average temperature were noticed after cutting the fourth and fifth ganglia: $0.3^{\circ} \mathrm{C}$ and $0.2^{\circ} \mathrm{C}$ respectively. The analysis of air temperature changes during the surgery confirmed that it was performed in stable air conditions (average air temperature changed less than $0.5^{\circ} \mathrm{C}$ ). The factor of air temperature stability is important not only from the medical point of view but specially in the aspect of proper interpretation of thermographic data.

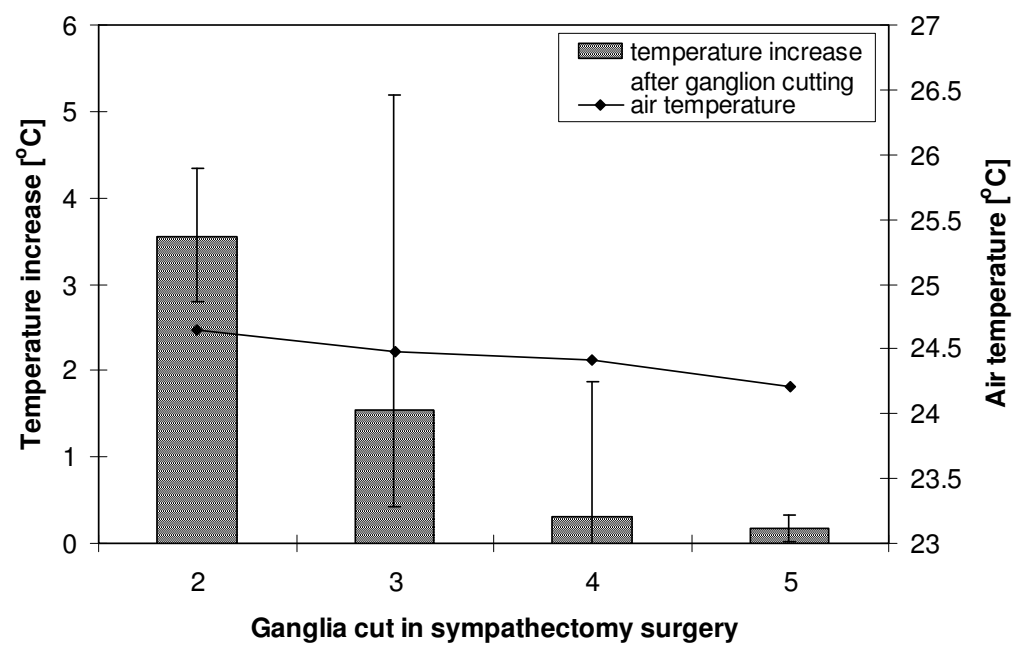

Fig. 5. Average temperature increase of the palm, range of temperature changes after cutting succeeding nerves and air temperature changes during surgery of one side. Order of ganglia cutting: 2nd, 3rd, 4th and 5th 


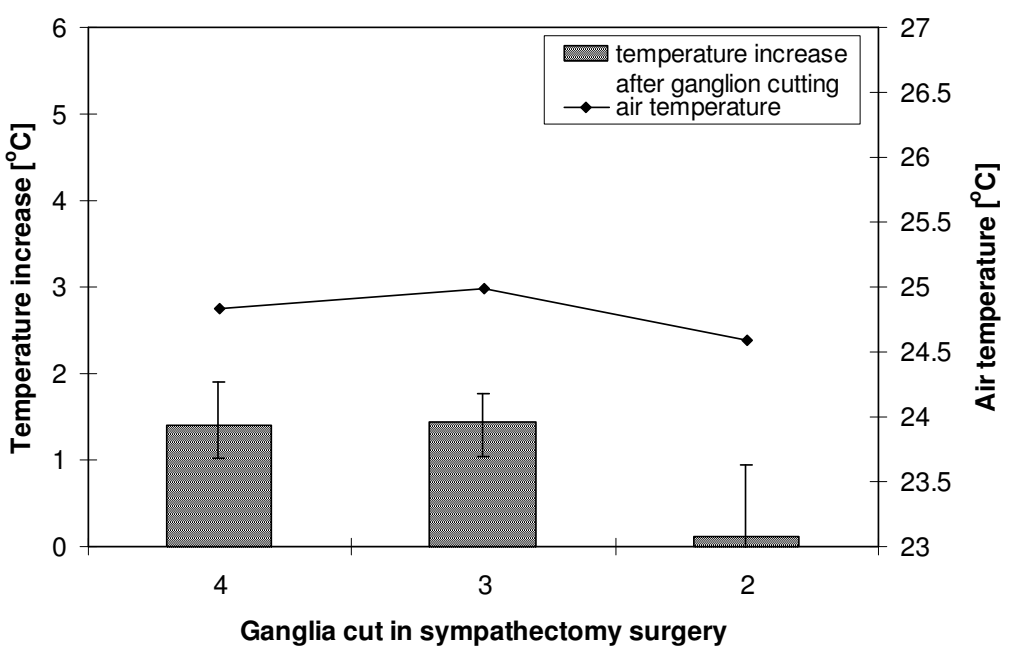

Fig. 6. Average temperature increase of the palm, range of temperature changes after cutting succeeding nerves and air temperature changes during surgery of one side. Order of ganglia cutting: 4th, 3rd and 2nd

In case of inverse order of ganglia cutting smaller increases of average palm temperature were observed. After cutting the fourth ganglion the average palm temperature from all examined patients increased by $1.5^{\circ} \mathrm{C}$, similarly as after cutting the third ganglion. The smallest palm temperature effect was observed after cutting the second ganglion. When this order of cutting was applied, the ranges of palm temperature changes for all patients were also much smaller. This results suggests that all three ganglia (II, III, IV) have strong influence on sweating intensity and in consequence on palm temperature distribution. Because the number of the studied patients in this preliminary examination was small it is difficult to evaluate the role of particular ganglia on final effect of sympatecthomy surgery. However, it is evident that thermographic method is a good tool for monitoring the intensity of oversweating in pre- and postsurgical stages. Rapid increase of temperature after cutting first two ganglia during operation gives information not only about the intensity of oversweating but also about effectiveness of applied surgery. Such surgical inspection can be a support for doctors in controlling the accuracy of treatment.

\section{Conclusions}

Thermographic examination is non-invasive method which occurred to be useful in detecting clinical oversweating.

Application of thermographic examination in postsurgery period confirms effectiveness of healing and enables to monitor possibility of diseases recurrence.

Considerable increase of palm temperature after thoracic ganglia cutting is a good quantitative indicator of operation effectiveness.

Because results come from preliminary stage of investigations the number of examined patients was not large. However, the homogeneity of this group due to identical type of disease and the same conditions of experiment confirm the reliability of obtained results.

\section{REFERENCES}

[1] N.A. Diakides, J.D. Bronzino. Medical Infrared Imaging, CRC Press, Taylor\&Francis Group, LLC (2008).

[2] N. Isogai, H. Kamiishi. Application of medical thermography to the diagnosis of Frey's syndrome. Head Neck. (1997) Mar;19(2):143-7.

[3] Y.C. Kim, J.H. Bahk, S.C. Lee, Y.W. Lee. Infrared thermographic imaging in the assessment of successful block on lumbar sympathetic ganglion. Yonsei Med J. (2003) Feb;44(1):119-24.

[4] S. Krüger, K.S. Fronek, M. Schmelz, T. Horbach, W. Hohenberger, C.H. Schick. Differential effects of surgical sympathetic block at the T2 and T4 level on vasoconstrictor function. : Clin Auton Res. (2003) Dec;13 Suppl 1:179-82.

[5] W.S. Rasband. ImageJ, U. S. National Institutes of Health, Bethesda, Maryland, USA, http://rsb.info.nih.gov/ij/, (1997-2007).

[6] J.A. Rosenblum, J.M. Cohen, M. Lee. Hyperhidrosis--a case history. Angiology. (1994) Jan;45(1):61-4.

[7] J.C. Tsai, K.B. Lim, S.Y. Lin, M.C. Kao. Thermographic study of palmar and facial skin temperature of hyperhidrosis patients before and after thoracic sympathectomy. J Formos Med Assoc. (2000) Jun;99(6):466-71. 
http://dx.doi.org/10.21611/qirt.2008.03_02_17 